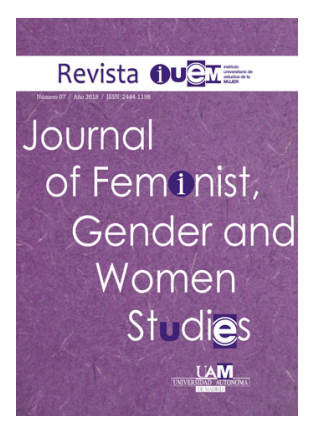

\title{
Discriminación interseccional: concepto y consecuencias en la incidencia de violencia sexual contra mujeres con discapacidad
}

\author{
Intersectional discrimination: concept and consequences for the prevalence of \\ sexual violence against women with disabilities
}

\author{
Alana Micaelle Cavalcante Carvalho, @ \\ ${ }^{1}$ Instituto Bartolomé de las Casas. España. \\ @ Autor/a de correspondencia: acavalca@inst.uc3m.es
}

\begin{abstract}
Resumen
El artículo examina las diferencias conceptuales entre las expresiones discriminación interseccional y discriminación múltiple, analizando por qué la primera refleja más adecuadamente la discriminación que enfrentan las mujeres con discapacidad. Pese a que muchas veces se utilicen como sinónimos, la idea de discriminación múltiple no enfatiza la interconexión entre las distintas formas de discriminación, que crean nuevas e intensificadas situaciones de opresión, por lo que no es la terminología que mejor explica ese proceso, mientras que la idea de discriminación interseccional permite visibilizar todas las formas de opresión y concienciar a la sociedad sobre ello. Considerando que las mujeres con discapacidad son un blanco para la discriminación destinada tanto a las mujeres como a las personas con discapacidad, comprender esa dinámica de opresión es fundamental para hacer frente a ella. Asimismo, para exponer la manifestación de ese debate teórico en la realidad, se ha optado por centrarse en la discriminación de las mujeres con discapacidad asociada a una las mayores expresiones de la vulneración de los derechos humanos s, que es la violencia sexual. Se ha elegido ese enfoque por la gravedad del delito, las especificidades de la violencia sexual cuando la víctima es una mujer con discapacidad -como tener al responsable de sus cuidados como agresor, no tener acceso a la educación sexual y enfrentar mayores barreras para denunciar y ser creída- y las consecuencias tras ser víctima de violación a que las demás mujeres no suelen estar sometidas, como la esterilización forzosa en caso de embarazo. Incluir a la discapacidad en los debates en torno a la violencia sexual contra las mujeres es importante para visibilizar esas especificidades y entender cómo la discriminación interseccional presenta consecuencias en ese ámbito.
\end{abstract}

Palabras clave: Discriminación interseccional, discapacidad, género, violencia sexual.

\begin{abstract}
The article examines the differences in meaning between the expressions intersectional discrimination and multiple discrimination. It analyzes why the former is more accurate to describe the discrimination suffered by women with disabilities than the latter. Even though both terms are commonly used as synonyms, the idea behind multiple discrimination does not emphasize the interconnexion among the numerous forms of discrimination, which create new and intensified forms of oppression. Consequently, the expression multiple discrimination is not the most suitable to explain this process, whereas the idea of intersectional discrimination underlines all forms of oppression and raise awareness among society. Considering that women with disabilities are a target for the discrimination suffered by both women and persons with disabilities, it is crucial to understand this oppressive dynamic to tackle it. Hence, to present a concrete manifestation of this theoretical debate, the article focuses on one of the strongest violations of human rights: sexual violence. This approach was chosen because of the seriousness of this felony and its nuances when the victim is a woman with disabilities - such as having a caregiver as the abuser, not having access to sexual education and facing increased barriers to report a crime and being believed - and the consequences imposed to the victim that, in general, other women do not face, such as compulsory sterilization. Including disability on the debates concerning sexual violence against women with disabilities is essential to highlight the referred nuances and to understand how intersectional discrimination present consequences on this matter.
\end{abstract}

Keywords: Intersectional discrimination, disability, gender, sexual violence. 


\section{INTRODUCCIÓN}

La discriminación que sufren las mujeres con discapacidad ha permanecido invisible por muchos siglos, pero hubo un significativo aumento de los debates acerca del tema durante las últimas décadas, especialmente después de la aprobación de la Convención sobre los derechos de las personas con discapacidad en 2006. Pese a esto, se observa que el asunto aún no ha sido suficientemente explorado y que tampoco el concepto es un consenso entre los teóricos, recibiendo distintos nombres: discriminación doble, múltiple o interseccional, entre otros.

El presente trabajo, que se enmarca en el enfoque de la discapacidad desde los derechos humanos, busca desmenuzar el concepto de discriminación interseccional y cómo esta impone consecuencias específicas en el riesgo específico que experimentan las mujeres con discapacidad de ser víctima de violencia sexual.

Para ello, se dividió el estudio en dos etapas: la primera dedicada a comprender el concepto de discriminación interseccional en contraste con la discriminación múltiple, muchas veces utilizadas como sinónimo a pesar de sus diferencias conceptuales, y las razones por las que, en este artículo, se entiende que la locución discriminación interseccional debería prevalecer sobre las demás.

La segunda se centra en los reflejos de la discriminación interseccional en la vulneración a los derechos sexuales de las mujeres con discapacidad, en concreto por medio de la violencia sexual. Se eligió enfocar en la violencia sexual, y no en las manifestaciones de vulneración de los derechos sexuales de modo general, por su gravedad y porque las mujeres con discapacidad no solo están expuestas a las amenazas de violencia sexual que sufren las demás mujeres, sino que también pueden vivir situaciones que estas no experimentan habitualmente y que pueden conllevar la violencia sexual, como la violación cometida por cuidadores o en instituciones de internamiento.

\section{EL CONCEPTO DE DISCRIMINACIÓN INTERSECCIONAL COMO HERRAMIENTA PARA EL ANÁLISIS DE LA DISCRIMINACIÓN QUE SUFREN LAS MUJERES CON DISCAPACIDAD}

Las mujeres con discapacidad enfrentan muchos desafíos en la sociedad y aún no han logrado ser tratadas con igualdad. La sociedad les impone una serie de estereotipos negativos que generan discriminación, como tratarlas como una carga, como eternas niñas o incapaces de acceder a la educación y al mercado de trabajo. Están sometidas, al mismo tiempo y de modo asociado, a la discriminación contras las mujeres y a la discriminación contra las personas con discapacidad, cuyas consecuencias pueden presentarse de distintos modos en la experiencia de las mujeres con discapacidad, como en una mayor dificultad de acceso al mercado de trabajo, de acceso a la justicia o la imposición de esterilización por parte de sus padres o representantes legales.

Se puede comprender la discriminación como "cualquier distinción, exclusión, restricción o preferencia basada en características como raza, color, sexo, lenguaje, religión, política u otra opinión, origen nacional o social, propiedad, nacimiento $u$ otro estatus, que tenga como propósito o efecto anular o impedir el reconocimiento, goce o ejercicio de los derechos y libertades por todas las personas, en igualdad de condiciones, de todos los derechos y libertades" (Makkonen, 2002: 4, traducción de la autora). La discriminación que tiene origen en dos o más elementos presentes en la misma persona ha recibido diversos nombres: discriminación doble, triple, múltiple, compuesta o interseccional. Sin embargo, se entiende en este artículo que, cuando estos dos o más elementos se interrelacionan profundizando la discriminación, se trata de discriminación interseccional ${ }^{1}$, como se desarrollará enseguida. La expresión discriminación interseccional fue desarrollada por la jurista Kimberlé Crenshaw (1989) basándose en los elementos género femenino y etnia negra. No obstante, actualmente es aplicable a cualesquiera dos o más identidades sociales discriminadas que se interrelacionan intrínsecamente en un individuo o grupo de individuos, como es el caso de mujer y discapacidad. Para comprender el significado y las especificidades de esa expresión, se expondrán a continuación las teorías de distintos autores sobre el asunto aplicándolas al contexto de las mujeres con discapacidad.

Según Platero (2014), la expresión discriminación interseccional se refiere a distintos orígenes estructurales de desigualdad $u$ organizadores sociales que establecen relaciones recíprocas, sobrepasando la noción intuitiva de doble o múltiple discriminación, ya que las identidades son construcciones dinámicas y conforman nuevas organizaciones sociales y desigualdades. Es lo que ocurre con la discriminación sufrida por las mujeres con discapacidad. Es decir, las barreras sociales y discriminatorias impuestas a ellas no son el resultado de una suma entre las discriminaciones enfrentadas por ser mujer y persona con discapacidad, sino que se profundizan, generando una discriminación aún más intensa y específica (González, 2011).

La Barbera (2015) sintetiza las novedades introducidas por la expresión discriminación interseccional en relación con otros términos, como discriminación múltiple: hallar el foco de atención en el individuo que se ubica en el cruce de los distintos sistemas de discriminación, que no pueden ser explicados de modo aislado; poner acento en el carácter simultaneo de los factores de discriminación y recalcar los efectos contraproducentes del análisis de un solo eje de discriminación.

Caballero (2016) concluye que la teoría de la interseccionalidad aplicada a las experiencias de las mujeres con discapacidad representa un elemento fundamental para desarrollar una base teórica que interrelaciona distintos elementos de estigmatización que, hasta entonces, eran invisibles en los ámbitos académico y activista. Como asevera Brah (2012:38): "aunque el concepto de interseccionalidad es

\footnotetext{
1 El término interseccional es interpretado por Bilge y Collins (2016) como una herramienta analítica para comprender y analizar la complejidad del mundo, en las personas y sus experiencias. Los hechos y condiciones de la vida social y política difícilmente pueden comprenderse a partir de un único elemento. En general, son influenciados por varios elementos que actúan conjuntamente, y la comprensión del término permite verlo de modo más evidente. No necesariamente la interseccionalidad posee una carga negativa, por el contrario, representa un paradigma y es inherente a todas las personas, no sólo aquellas que enfrentan más de una forma de discriminación, ya que cada individuo se identifica con una categoría referente al sexo, etnia, edad, nacionalidad, entre otros (Serra, 2017).
} 
muy reciente, los procesos de interseccionalidad han operado desde hace mucho tiempo".

La discriminación interseccional se manifiesta en el contexto de discriminación estructural vigente, que representa la identificación de desigualdades de derecho y de hecho derivadas de una situación de exclusión social o de sometimiento de ciertos grupos sociales -como las mujeres y las personas con discapacidad-por otros-como los hombres y las personas sin discapacidad-, en virtud de prácticas sociales, culturales y prejuicios históricos excluyentes (Pelletier, 2014). La discriminación interseccional que sufren las mujeres con discapacidad se manifiesta de distintos modos, entre ellos la intensificación, desde el punto de vista social, de las características de fragilidad femenina asociadas a ellas. Asimismo, suelen ser más infantilizadas y sobreprotegidas por sus familias que las demás mujeres y que los hombres con discapacidad (Carvalho et al, 2014). Además, mientras que a las mujeres sin discapacidad socialmente se les impone un rol de madre y esposa -y ellas reivindican romper con ese tipo de estereotipo- a las mujeres con discapacidad se les deniega tanto los roles negados a las demás mujeres como los que podrían llamarse estereotípicamente femeninos (Carrasco et al, 2006).

Hanna y Rogovsky (2008) teorizan sobre los tres aspectos del sistema sociocultural en vigor más relevantes para comprender la discriminación interseccional ${ }^{2}$ que enfrentan las mujeres con discapacidad: el sexismo, la discriminación contra personas con discapacidad y lo que denominan factor adicional. El factor adicional es, en realidad, un conjunto de factores que afecta especialmente a ese colectivo, a saber: la inadecuación social debido a la discapacidad, que hace que la persona con discapacidad no esté totalmente incluida en la sociedad; el cuidado de las personas con discapacidad, comúnmente atribuido a las mujeres $y$, en el caso de que sea la mujer quien posee un discapacidad, se considera que no está habilitada para cumplir su rol social y la belleza robada, que considera las mujeres con discapacidad inadecuadas a los roles de símbolo sexual y pareja (Hanna y Rogovsky, 2008). Desde esa óptica, se entiende que ellas enfrentan la presión social y discriminación debido primordialmente a estos elementos que, relacionados, generan consecuencias percibidas de modo más intenso por las mujeres con discapacidad -al compararse con los hombres con discapacidad o con las demás mujeres- incluso en el ámbito de los derechos sexuales y reproductivos, como son ejemplo de ello el aborto no consentido y la esterilización forzosa (Ballester, 2013).

En definitiva, al contemplar las vidas de las mujeres con discapacidad y pensar políticas públicas destinadas a ellas con el objetivo de promover la igualdad, no cabe plantear si su factor determinante es el género o la discapacidad, pues sus experiencias como individuo no son fragmentadas (González, 2011), sino que mezclan ambos elementos de modo intrínseco, no pudiendo, el uno o el otro, ser desconsiderados al analizar la condición social de la mujer

2 En su investigación, los autores no utilizan la expresión discriminación interseccional. Ellos se refieren a una suma de discriminaciones y un conjunto de factores de discriminación que resultan en relaciones complejas entre sí. Sin embargo, en el presente trabajo se entiende que esa idea se adecua al concepto de discriminación interseccional aquí utilizado. Véase: Hanna y Rogovsky, 2008. con discapacidad o los orígenes de la discriminación que sufren. Como sostiene Jenny Morris (1996:19), "no creo que, a la hora de contemplar nuestra vida, tenga ninguna utilidad plantear la cuestión de si el determinante más importante de la experiencia es 'el género o la discapacidad'. [...] En parte, pienso así porque nuestras experiencias no están fragmentadas en teorías analíticas".

En ese sentido, el objetivo de comprender el concepto de interseccionalidad no puede ser comparar distintas formas de opresión y colectivos discriminados preguntándose quien es más oprimido por la sociedad y buscando una respuesta única (Eibach y Purdie-Vaughns, 2008). Por el contrario, la teoría de la discriminación interseccional pretende dar relevancia a las distintas manifestaciones de discriminación que se impongan sobre el individuo, impidiendo que alguna de las formas de discriminación se invisibilice. Muchas veces la discriminación viene asociada a aspectos de la vida considerados estructurales o inmutables, y eso dificulta identificar la manifestación de la discriminación, al ser socialmente tratada como algo intrínseco a la situación o naturalizada. Para solucionar esto, Crenshaw (2002) propone que las dimensiones estructurales que pueden ser motivo de discriminación -como el género y la discapacidadsean siempre consideradas en el primer plano de análisis ${ }^{3}$. Para ello, se puede aplicar el método 'haz otra pregunta', propuesto por Matsuda (1991:1184): “al identificar actitudes racistas, debemos preguntarnos '¿dónde está el patriarcado en eso? Cuando veamos algo que parece machista, preguntarnos, ‘¿dónde está el heterosexismo en esto?"” Observar situaciones discriminatorias desde una perspectiva interseccional permite identificar las causas de discriminación obvias y no obvias, contribuyendo a no preterir ninguna de sus manifestaciones.

Las mujeres con discapacidad, al poseer más de una identidad socialmente considerada inferior, experimentan invisibilidad interseccional por no corresponder al prototipo de los grupos a los que pertenece, el de mujeres y de personas con discapacidad, y, en consecuencia, no ser plenamente reconocidas como miembros de estos grupos. Los hombres son tratados como el estándar neutral, la norma en la sociedad, mientras que las mujeres son consideradas un desvío a esa norma, la experiencia femenina es "el otro" (Eibach y Purdie-Vaughns, 2008). Análogamente, se considera a las personas con discapacidad como fuera de los estándares capacitistas de la sociedad, una excepción a la regla general.

A su vez, Harnois e Ifatunji (2011) resaltan que hay una dimensión espacial de la discriminación, determinada por jerarquías de raza, género, clase, sexualidad $-y$, cabe añadirlo, capacidades. Asimismo, la posibilidad de sufrir discriminación y la forma como esta se presenta depende en parte del ambiente donde el individuo está. En el caso de las personas con discapacidad, se observa una relación directa entre las barreras existentes en un espacio social -no solo de carácter arquitectónico, sino también actitudinal y cognitivoy la discriminación contra las personas con discapacidad en

3 La autora presenta como ejemplo la experiencia de las mujeres dalit (intocables) en India, que sufren violencia en espacios públicos por parte de miembros de castas más altas al realizar actividades de responsabilidad de las mujeres, como coger agua en una fuente. Ese tipo de violencia es habitualmente descrito como una discriminación de castas, ignorando el componente de género en la cuestión (Crenshaw, 2002). 
estos espacios. Una situación que ilustra la dimensión espacial de la discriminación sufrida por las mujeres con discapacidad es la dificultad aumentada que enfrentan para ir al ginecólogo y demás servicios de atención a la salud. Como consta en la Observación General 3: mujeres y niñas con discapacidad, de 2016, del Comité sobre los derechos de las personas con discapacidad de Naciones Unidas (en adelante, Comité): “EI equipo y las instalaciones de atención de la salud, incluidos los aparatos de mamografía y las camillas para exámenes ginecológicos, suelen ser físicamente inaccesibles para las mujeres con discapacidad. [...] Las barreras debidas a la actitud del personal de atención de la salud y el personal conexo pueden dar lugar a que se deniegue a las mujeres con discapacidad el acceso a servicios y profesionales de atención de la salud".

Los distintos abordajes teóricos sobre la discriminación interseccional permiten delinear el sentido de la expresión, sus usos y posibilidades. En definitiva, se utiliza la locución discriminación interseccional para referirse a dos o más causas de discriminación que poseen consecuencias más intensas y específicas que un análisis de cada eje de discriminación por separado podría revelar. La idea de utilizar esa expresión sobrepasa la noción de que algunas de las causas de discriminación pueden relacionarse, evidenciando las distintas causas de discriminación como igualmente relevantes y hallándolas en el primer plano de análisis, lo que permite que la identificación de las posibles relaciones entre diferentes causas de discriminación ocurra de modo mucho más inmediato.

Otro elemento fundamental de la discriminación interseccional es la simultaneidad. El esfuerzo teórico de subdividir y fragmentar procesos discriminatorios que un individuo experimenta de modo asociado no refleja la realidad. Cuando una mujer con discapacidad sufre discriminación, no siempre es posible determinar si lo que motiva la discriminación es el género o la discapacidad o si el primero no tiene ninguna influencia en discriminaciones en las que predomina el segundo. Asimismo, tener presente la simultaneidad de las manifestaciones de discriminación hace más fácil percibir si hay más de una causa de discriminación reflejada en determinados hechos.

En relación con el lenguaje más adecuado para denominar ese proceso que afecta a las mujeres con discapacidad, la expresión discriminación interseccional no es la única utilizada para describirlo, siendo común ver el uso de otras como discriminación múltiple, frecuentemente utilizada por el Comité sobre los derechos de las personas con discapacidad. Se identifica también una difusión desigual de la expresión desde la perspectiva geográfica. En el pensamiento anglosajón ha prevalecido el uso de discriminación interseccional, mientras que en Europa continental esa locución no ha sido tan explorada, predominando el uso de discriminación múltiple (La Barbera, 2015). Ante esa dualidad, ha surgido la necesidad de justificar teóricamente por qué la primera expresión debería prevalecer en lugar de las demás.

Crenshaw (2002) esclarece que la noción de interseccionalidad busca reflejar las consecuencias de la interacción entre dos o más formas de discriminación, lo que sobrepasa una simple yuxtaposición de una multitud de discriminaciones. Esto no quiere decir que los conceptos de doble o múltiple discriminación necesariamente desconsideren la interrelación entre dos o más formas de discriminación ${ }^{4}$, sino que el nombre no da relieve a esa perspectiva, que debe estar en un primer plano. La expresión discriminación interseccional, a su vez, recuerda constantemente la dinámica de ese proceso de interacción entre los distintos ejes de discriminación.

Permitir que se olvide que la relación entre más de una fuente de discriminación puede generar desdoblamientos nuevos y únicos impide una comprensión integral de ese tipo de discriminación. Como desarrolla Crenshaw (2002:177, traducción de la autora) en relación con las mujeres que no pertenecen a la etnia blanca: "La importancia de desarrollar una perspectiva que destaque y analice la discriminación interseccional está no solo en el valor de las descripciones más precisas sobre las experiencias vividas por las mujeres racializadas, sino también en el hecho de que las intervenciones basadas en comprensiones parciales o incluso distorsionadas de las condiciones de las mujeres son, seguramente, ineficientes y quizás contraproducentes. Solo con un análisis más a fondo de las dinámicas variables que conforman la subordinación de las mujeres racialmente marcadas se pueden desarrollar intervenciones y protecciones más eficaces".

En la literatura académica sobre la discriminación, muchas veces se observa el uso de las expresiones discriminación múltiple y discriminación interseccional como si fuesen sinónimos, lo que Makkonen (2002) denomina desorganización conceptual. El uso indiferenciado de esas expresiones hace que algunos autores ignoren la importancia de reforzar la interrelación entre los distintos focos de discriminación o incluso admitan conocer las diferencias entre estos conceptos, pero opten por utilizarlos como sinónimos por razones operativas ${ }^{5}$. Esto, además de invisibilizar el objetivo del uso de la expresión discriminación interseccional, es contraproducente para una comprensión adecuada de la discriminación que sufren las mujeres con discapacidad. Como afirman Caballero y Vales (2012), nadie es solamente mujer o persona con discapacidad, por lo que conocer esas intersecciones es fundamental para evitar que la discapacidad invisibilice otras dimensiones del sujeto y se perciba sólo a individuos con discapacidad, sin identidad sexual o identidad de género. Las diferencias importan, y el uso indiferenciado de las expresiones discriminación múltiple y discriminación interseccional desconsidera eso.

Debido a esa desorganización conceptual, la diferencia semántica entre la discriminación interseccional y múltiple es fluida. Algunos autores acercan su sentido al de la discriminación interseccional, mientras que otros entienden ambos conceptos de modo independiente, como en el presente artículo. Lama (2013) define la discriminación múltiple como la que se produce por la concurrencia de varios motivos al mismo tiempo. A su vez, en la Observación General sobre mujeres y niñas con discapacidad el Comité de la ONU utiliza discriminación múltiple para hacer referencia a una situación en la que un individuo experimenta dos o más

\footnotetext{
4 Rey (2008) entiende que no se puede comprender la discriminación múltiple separadamente a la idea de que ambos motivos de discriminación operan simultáneamente, lo que se acerca al concepto de discriminación interseccional.
}

5 Véase: Gomiz, 2016. 
motivos de discriminación y discriminación interseccional para referirse a una situación en la que varios motivos interactúan al mismo tiempo de forma inseparable.

A su vez, Makkonen (2002) es uno de los teóricos que dio mayor relieve a la importancia de la comprensión y del uso de la idea de discriminación interseccional. No obstante, el autor defiende el uso simultáneo de las expresiones discriminación múltiple, compuesta e interseccional para distintos tipos de discriminación, según el contexto. Para Makkonen, se está ante una discriminación múltiple cuando un individuo sufre discriminación por razones distintas en momentos distintos, utilizando como ejemplo una mujer con discapacidad que sufre discriminación en su trabajo debido a su género y en un edificio público no accesible debido a su discapacidad. Por otro lado, se trataría de discriminación interseccional en sentido restrictivo (en oposición al sentido amplio, que englobaría bajo una única expresión las distintas formas de discriminación propuestas), cuando, en una situación en la que ocurre discriminación, distintos orígenes de discriminación interactúan. Sin embargo, estar de acuerdo con esa visión significa permitir fragmentar y analizar separadamente procesos que, en realidad, ocurren de modo interrelacionado. Preestablecer que una mujer con discapacidad sufre discriminación en su trabajo por razón de género y disociar esa discriminación de la discapacidad sin antes analizar los hechos permite que ciertas formas de discriminación no obvias permanezcan invisibles. Pese a que el autor posteriormente aclare que el hecho de que una persona sufra discriminación múltiple no impide que sufra también discriminación interseccional, su propuesta de compartimentar la discriminación basada en más de una razón en distintas categorías no deja eso claro.

Utilizar la expresión discriminación interseccional en vez de discriminación múltiple debe ser considerada un compromiso con la difusión de la idea de interrelación entre las distintas formas de discriminación, reforzando que no pueden ser interpretadas separadamente. Como afirma Makkonen (2002:58): “El beneficio más importante de un enfoque interseccional es la habilidad de desvelar formas y manifestaciones de discriminación anteriormente no identificadas. El análisis interseccional también permite detectar distintos contextos y otros factores que han contribuido para tal discriminación y desventaja, y una comprensión de las distintas formas y contextos de la discriminación y desventaja claramente ofrecen una mejor plataforma para la acción política y legal centrada en combatir la discriminación" (traducción de la autora). Sin embargo, esa posición no es unánime. En efecto, Rey (2013) también critica el uso indiferenciado de las expresiones múltiple e interseccional, pero su solución difiere de la aquí defendida: propone elegir la nomenclatura discriminación múltiple, por su uso difundido en las instituciones europeas, su reconocimiento en el diccionario y por ser más expresiva en español. No cabe argumentar cuanto a la mayor o menor expresividad de un término, algo subjetivo. Tampoco parece acertado preterir el uso de la expresión discriminación interseccional debido a su ausencia en el diccionario, una herramienta que recoge el uso de las palabras y que, en consecuencia, puede incorporar nuevos vocablos. Por último, optar por el uso de esa nomenclatura por ser la más común en los documentos de la Unión Europea no parece adecuado.
Cualquier término está susceptible a actualizaciones, especialmente cuando se identifica que la propuesta más reciente está más acorde con la idea expresada. Asimismo, en los últimos años la Unión Europea ha hecho mención expresa a la discriminación interseccional, como en la resolución de 2011 sobre igualdad entre mujeres y hombres al citar las mujeres de las minorías, en la resolución de 2013 sobre los aspectos de género de las estrategias de inclusión de las mujeres de etnia Romaní y en el informe de 2016 sobre discriminación interseccional, igualdad de género y leyes antidiscriminación en la Unión Europea.

No se quiere con esto decir que la expresión discriminación múltiple es totalmente inadecuada o que debería ser excluida definitivamente de los estudios sobre más de una fuente de discriminación. Sin embargo, es importante recordar el valor simbólico del lenguaje (Lledó, 2005) y su intencionalidad, por lo que nombrar precisamente situaciones de discriminación permite enfrentarlas de modo más específico (Harpur, 2009).

\section{LOS RIESGOS ESPECÍFICOS DE SUFRIR VIOLENCIA SEXUAL COMO MANIFESTACIÓN DE LA DISCRIMINACIÓN INTERSECCIONAL CONTRA MUJERES CON DISCAPACIDAD}

La sociedad contemporánea no sabe convivir con las diferencias, no valora la diversidad y considera la ausencia de discapacidad como el estándar, por lo que Pié y Riu (2014) la clasifican como "anormalfóbica". Debido a esa aversión por la diversidad, se construyen imágenes sociales negativas de las mujeres con discapacidad como inferiores, incompletas o menos mujeres, y esa manifestación de la discriminación interseccional presenta consecuencias en distintos ámbitos, entre ellos el de los derechos sexuales.

Tras comprender cómo la discriminación interseccional opera y la importancia de ese concepto, se desmenuzarán a continuación los desdoblamientos de esa discriminación en uno de los ámbitos esenciales de la vida de las mujeres con discapacidad y, paradójicamente, uno de los que más ha sufrido vulneraciones a lo largo de la Historia: el derecho a gozar de su sexualidad y de una vida libre de violencia desde la perspectiva de una de las mayores vulneraciones de derechos en ese ámbito, que es la violencia sexual. Barreras arquitectónicas, actitudinales y mitos socialmente construidos en torno a las mujeres con discapacidad impiden que ellas puedan gozar y ejercer plenamente sus derechos sexuales (Gesser y Nuernberg, 2014), pese a que sea un derecho reconocido a las personas con discapacidad ${ }^{6}$, y las convierten en un blanco potencial para la violencia sexual.

Es importante tener presente qué son los derechos sexuales y por qué la violencia sexual representa una vulneración de ellos. No hay una definición internacional

\footnotetext{
6 En la Convención sobre los derechos de las personas con discapacidad de Naciones Unidas (en adelante, Convención), se hace una referencia al tema en el artículo 25, dedicado a la salud, mencionando la obligación de los Estados partes de elaborar programas de atención a la salud sexual y reproductiva. En el artículo 23, sobre el respeto al hogar y a la familia, se rechaza la discriminación en asuntos relacionados con el matrimonio, la familia, la maternidad y paternidad y las relaciones personales, además de que se debe tener acceso a información, educación sobre reproducción y planificación familiar y el derecho a mantener su fertilidad. A su vez, la Observación General sobre mujeres y niñas con discapacidad menciona expresamente los derechos sexuales y reproductivos.
} 
o constitucionalmente reconocida en España para los derechos sexuales, pero es posible inferir su amplitud a partir de su aplicación. El derecho a la sexualidad incluye la protección a la identidad sexual, la orientación sexual, la elección de pareja, la educación e información sexual y la ausencia de actividad sexual coercitiva (Villanueva, 2006). Complementando esa idea, Moya (2009) entiende que los derechos sexuales se relacionan con la libertad sexual, la salud sexual, la autonomía, la privacidad, el placer y la libertad sobre el cuerpo. A su vez, las bases de los derechos reproductivos fueron establecidas en la Plataforma de Acción de la Cuarta Conferencia Mundial sobre la Mujer, en 1995: "El reconocimiento del derecho básico de todas las parejas $e$ individuos a decidir libre y responsablemente el número de hijos, el espaciamiento de los nacimientos y el intervalo entre éstos y a disponer de la información y de los medios para ello y el derecho a alcanzar el nivel más elevado de salud sexual y reproductiva. También incluye su derecho a adoptar decisiones relativas a la reproducción sin sufrir discriminación, coacciones ni violencia, de conformidad con lo establecido en los documentos de derechos humanos".

La violencia sexual7, al ser la expresión máxima de la objetificación del cuerpo femenino y de la nulidad de la voluntad de la mujer, rompe con estos derechos contemplados en el espectro de los derechos sexuales, afectando su derecho a vivir una vida libre de violencia, a la autodeterminación sexual, a la salud, la integridad física y la dignidad humana. La mujer víctima de violencia sexual sufre, por el hecho de ser mujer, coacción, violencia, amenaza a su integridad y salud física y mental y imposibilidad de elegir con quien desea mantener relaciones sexuales.

Asimismo, ese tipo de violencia refleja la desigualdad de poder entre hombres y mujeres en la sociedad, siendo una consecuencia de la sociedad machista y patriarcal ${ }^{8}$ y que refleja la estructura social de subordinación de la mujer ante el hombre (Segato, 2017). Beneficiándose de su posición de dominación sobre las mujeres, los hombres ejercen una violencia sobre ellas que puede manifestarse de distintos modos, ${ }^{9}$ entre ellos el de la violencia sexual (Conde, Mun y Portillo, 2006).

La violencia sexual es una amenaza constante para todas las mujeres, con discapacidad o no. No hay ninguna sociedad, en la actualidad, que esté libre de ese delito, que es una de las mayores demostraciones de violación de los derechos humanos, afectando al plan de vida de las víctimas y generando consecuencias físicas, psicológicas y sociales (Basile y Smith, 2011). La violencia sexual puede ocurrir en cualquier contexto, como en una relación de pareja, en el ambiente laboral o educativo, en la calle o durante guerras y crisis humanitarias (Organización Mundial de la Salud, 2013).

De modo general, las víctimas de violencia sexual sufren coacción, violencia física, amenaza o intimidación por parte del agresor para que este pueda realizar el acto

\footnotetext{
7 La Organización Mundial de Salud (OMS), define la violencia sexual como "todo acto sexual, la tentativa de consumar un acto sexual, los comentarios o insinuaciones sexuales no deseados, o las acciones para comercializar o utilizar de cualquier otro modo la sexualidad de una persona mediante coacción por otra persona, independientemente de la relación de esta con la víctima, en cualquier ámbito, incluidos el hogar y el lugar de trabajo".

8 Véase: Cobo, 2011.
}

9 Véase: Conde, Mun y Portillo, 2006. delictivo y sienten miedo a denunciar y no ser creídas o ser revictimizadas (Lodrick y Mason, 2013). Además, socialmente se considera la violencia sexual un hecho vergonzoso para la víctima y muchas siguen siendo estigmatizadas, lo que también dificulta las denuncias (Dartnall y Jerkes, 2013). Cuando deciden denunciar o contar a alguien el suceso, sus interlocutores pueden reaccionar con incredulidad, distorsionar sus palabras o considerarlas mentirosas (Hlavka, 2014).

Hay diversos factores que conllevan la prevalencia de violencia sexual, como la existencia de una norma social tradicional basada en la superioridad masculina; la naturalización de la violencia, la objetificación del cuerpo femenino, la sensación de impunidad del agresor y la creencia en el uso de la fuerza (Casique y Furegato, 2006).

Previamente a analizar las especificidades y profundizaciones asociadas a esos factores en caso de que la víctima sea una mujer con discapacidad, es relevante comprender la dimensión del problema por medio de los índices de violencia sexual que sufren. La escasez de estadísticas fiables en escala global revela la invisibilidad social y académica de la violencia sexual contextualizada a la discapacidad. Datos contrastantes, ausencia de comparación entre los datos de violencia sexual contra mujeres con discapacidad y las demás mujeres y carencia de una metodología clara son algunas de las problemáticas identificadas al buscar estadísticas sobre el asunto. Sin embargo, España dispone de datos de esa naturaleza por la Macroencuesta de violencia contra la mujer 2015, utilizada por la Fundación Cermi Mujeres para elaborar el Informe sobre violencia de género hacia las mujeres con discapacidad en 2016. La macroencuesta fue realizada con 10.171 mujeres, entre ellas 442 con discapacidad. En la comparación de las situaciones de violencia sexual padecida por mujeres con discapacidad y sin discapacidad por la pareja actual o su anterior pareja, un $8,5 \%$ de las mujeres con discapacidad declaró haberla sufrido, frente a un 3,9\% de las demás mujeres. Al incluir datos referentes a la violencia sexual fuera del contexto de la pareja o expareja, un $7 \%$ de las mujeres con discapacidad declaró haber sido víctimas de violencia sexual. Sumando a la violencia sexual la violencia física, un $35,1 \%$ de las mujeres con discapacidad afirmó haberla sufrido, cometida por su pareja, expareja o una persona desconocida, mientras que un $23,7 \%$ de las demás mujeres declaró lo mismo.

¿Por qué, al menos en España, las mujeres con discapacidad sufren una mayor incidencia de violencia sexual? No hay una respuesta única a ese planteamiento, sino un conjunto de elementos que permiten comprender cómo el agresor ve a la mujer con discapacidad y las consecuencias de esa visión para la violencia sexual, que serán analizados a continuación. En la literatura, no es raro ver un rol de las causas que conllevan las mujeres con discapacidad a sufrir violencia sexual que no contemplan la única condición necesaria para que haya violencia sexual: la existencia de un individuo con ánimo de agredir sexualmente ${ }^{10}$. Se centran en elementos como el ambiente de la acometida de violación, la sobreprotección familiar y la dificultad de acceso a la justicia, aspectos cruciales para entender el contexto en el que ocurre

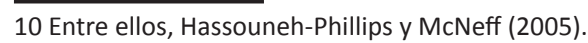


la violencia contra la mujer con discapacidad y los matices que diferencian ese tipo de violencia sexual de aquella que sufren las demás mujeres, pero que no son la causa de la violencia. Tener eso presente es crucial para entender la premisa de este trabajo.

Los elementos que permiten comprender por qué las mujeres con discapacidad son un blanco para la violencia sexual poseen relación directa con la imagen negativa que el agresor construye de la mujer con discapacidad y también con aquellos previamente mencionados como determinantes para la incidencia de violencia sexual contra las mujeres en general, y entre ellos están:

l) la visión estereotipada sobre las mujeres con discapacidad, que le hacen creer que son incapaces de tomar decisiones o de presentar una denuncia y ser creídas (Díaz, 2013), lo que puede conllevar el agresor a intentar desacreditar la historia de la víctima, dificultando que la mujer con discapacidad sea creída (Gobierno Vasco, 2012) y originándole una sensación de impunidad;

II) la percepción de que ellas oponen menos resistencia a la violencia (Junta de Andalucía, sin fecha). Elman (2005) afirma que los violadores suelen elegir sus víctimas buscando aquellas que creen más indefensas. Ante una mujer con discapacidad, el agresor puede suponer que ella se adecua a esas características, debido a la imagen victimizada que la sociedad construye en torno a las personas con discapacidad (Arnau, 2005);

III) la percepción del cuerpo de la mujer con discapacidad como una "mercancía dañada". Caballero (2016) señala que, socialmente, los casos de violencia sexual cuyas víctimas son mujeres con discapacidad se consideran un ataque a un bien jurídico de menor valor por el mito de que su cuerpo es algo imperfecto que puede ser agredido y mutilado. Esto se relaciona directamente con la objetificación del cuerpo femenino, lo que banaliza la violencia (Almeida, 2016);

IV) la posición de autoridad y cuidado que puede ejercer sobre la víctima y el aislamiento y la exclusión social que experimentan algunas mujeres con discapacidad, como las que viven en instituciones (Ramiro, 2005). Si una mujer con discapacidad vive en instituciones de salud, sus referentes masculinos no se restringen a personas de su círculo familiar y social, incluyendo también a cuidadores y personal sanitario. Muchos realizan actividades que exigen un contacto íntimo, como ducharla y vestirla, además de ejercer una relación de poder y jerarquía sobre la víctima, y se valen de eso para la acometida del delito (Gobierno Vasco, 2012). Muchas víctimas sienten miedo a denunciar y con ello romper con el contacto social o los cuidados que recibe del agresor (López, 2007);

V) la sobreprotección familiar, tratándolas como niñas (Benedet y Grant, 2014), controlando sus cuerpos con discursos y actitudes de rechazo ante la intención de las mujeres de practicar actividades sexuales (Cruz, 2012) y la denegación de educación sexual (Gesser y Nuernberg, 2014). Las familias tratan a las mujeres con discapacidad como si no fuesen adultas, negándoles a salir de noche, tener pareja o vivir su sexualidad y disponer de su propio cuerpo. Eso les dificulta reconocer qué es apropiado en una relación consentida y les dejan menos oportunidades de aprender qué les gusta o no sexualmente y establecer límites en cuanto a eso (Howland et al, 2001). Socialmente, el ejercicio de la sexualidad es considerado un privilegio, no un derecho, no siendo reconocido para aquellos que han estado marginados en la sociedad. Eso es una realidad en el caso de las mujeres y se intensifica al tratarse de mujeres con discapacidad. En consecuencia, las familias y los profesionales en contacto con la mujer con discapacidad no reconocen su actitud represiva como una limitación a los derechos de la mujer, encubriéndola bajo la excusa de protección (Mogollón, 2014). Además, algunos estudios indican que, de modo general, las familias de hombres con discapacidad se muestran más dispuestas a que estos ejerzan su interés erótico-sexual en relaciones de pareja, al contrario de lo que ocurre con las mujeres (Cruz, 2004).

VI) La denegación de educación sexual de parte de las familias, educadores y profesionales sanitarios. La denegación de educación sexual intenta evitar el interés sobre el asunto e impedir que ellas sean sexualmente activas, pues hay un mito de que la educación sexual despierta su sexualidad inocente y dormida. Igualmente, denegar educación sexual a la mujer con discapacidad impide el goce de una serie de derechos sexuales, como el derecho a la intimidad, a recibir información, a relacionarse, a explorar su cuerpo y a ser concienciada sobre la violencia sexual (Campo, 2003). Pese a que muchas mujeres con discapacidad hayan sido expuestas a profesionales de la salud a lo largo de toda su vida, estos profesionales frecuentemente las atienden sin ofrecerles información sexual (Cruz, 2004). Además, cuando se dialoga sobre sexualidad, se suele centrar en temas como el reproche a actitudes consideradas inadecuadas, la prevención de abusos sexuales o asuntos de carácter fisiológico, sin una preocupación por abordar el placer o una vida sexual sana (Gesser y Nuernberg, 2014). También se asocian a ellas mitos sociales de carácter diametralmente opuesto, como que son hipersexualizadas, presentando deseos incontrolables y exagerados, o que son seres asexuados, deshumanizándolas.

VII) la dificultad aumentada al intentar denunciar el agresor (Díaz, 2013), debido a las barreras ${ }^{11}$ de acceder a la justicia (Carignano y Palacios, 2012). Los obstáculos para denunciar una violación y ser creída son enfrentados por las mujeres de modo general, pero, al tratarse de mujeres con discapacidad, esa dificultad se intensifica. Esto ocurre por la intersección entre lo que puede ocurrir con las mujeres independientemente de su discapacidad-como la presunción de que está inventando el delito (Barros, Jorge-Birol, 2007)con construcciones sociales negativas más asociadas a las mujeres con discapacidad, como la idea de que no pueden expresarse de modo coherente (Díaz, 2013). Igualmente, una de las dificultades de las mujeres con discapacidad de denunciar proviene del apoyo que necesitan para hacerlo, lo que se convierte en un problema cuando su interlocutor no trata la violencia sufrida como un delito, la minimiza, o considera mejor no exponer a la víctima a un proceso judicial, desincentivándola a denunciar. Estas actitudes contribuyen a la disminución de los índices de denuncias, la impunidad de los agresores y son un incentivo para que se sigan cometiendo delitos de carácter sexual.

Además de los argumentos previamente mencionados,

11 Véase: Carignano y Palacios, 2012. 
algunos autores ${ }^{12}$ señalan factores para el riesgo de violencia sexual que se asocian a las propias mujeres con discapacidad, como el tipo de discapacidad, su nivel de autonomía o autoestima. No obstante, aunque estos elementos puedan influenciar la percepción que el agresor tiene de la víctima, enfatizar aspectos individuales singulariza las causas de violencia sexual, disminuye el peso del real culpable de la violación -el agresor- y refuerza estereotipos perjudiciales al combate a la violencia sexual, como la marginalización y culpabilización de la víctima (Mays, 2006). Para romper con eso, Arenas (2015) propone desviar el foco de causas individuales de la violencia y centrarlo en condiciones históricas y sociales que refuerzan la discriminación y contribuyen a reforzar y perpetuar la violencia. Si el feminismo logró que la violencia sexual dejara de ser considerada una cuestión privada y coyuntural, independiente de la ropa o del comportamiento de la víctima, las teorías de análisis de la violencia contra las mujeres con discapacidad también pueden lograrlo.

Las consecuencias de la violencia sexual también presentan especificidades cuando la víctima es una mujer con discapacidad. Entre ellas están el aborto y la esterilización realizados de modo forzoso cuando de la violación resulta un embarazo, sin una preocupación por el deseo de la mujer en caso de haber sido incapacitada judicialmente. Una mujer sin discapacidad tiene derecho a elegir sobre realizar o no un aborto de un embarazo derivado de una violación en España. A las mujeres con discapacidad incapacitadas judicialmente, en contraste, se les deniega el derecho a elegir sobre su vida sexual y reproductiva con apoyos (Cruz, 2012) y, al ser una práctica legal y socialmente aceptada, no se entiende como una violencia.

La esterilización forzosa a mujeres con discapacidad intelectual es común y legal en España. Para que se pueda hacerla, es necesario someterla a un proceso de incapacitación judicial, procedimiento que, hasta el momento, no se ha adecuado a las directrices de la Convención ${ }^{13}$ y refleja una configuración jurídica basada en un enfoque médicorehabilitador $^{14}$ (Cuenca, 2012).

Esterilizar a una mujer con discapacidad contrariamente a su voluntad significa no solo denegarle la posibilidad de ser madre, en caso de que ese sea su deseo, sino también el derecho a la autodeterminación de su propio cuerpo, a la integridad física, a la libertad y a la no discriminación. Es un ejemplo evidente de la discriminación interseccional que sufren las mujeres con discapacidad, pues representa una demostración de discriminación a la que no suelen estar expuestos los hombres con discapacidad o las demás mujeres (Makkonen, 2002), además de una práctica legitimada por muchos Estados y por la sociedad basándose en distintos argumentos, entre ellos la prevención de abusos sexuales (Serra, 2017). Sin embargo, esterilizar para prevenir abusos sexuales es incoherente y contraproducente, pues puede mascarar la violencia sexual (Bujan, 2016). Además, cabe resaltar que la esterilización forzosa es utilizada como instrumento para reprimir el derecho de la mujer con discapacidad a decidir sobre su propio cuerpo y sobre

12 Véase: Brownridge, 2006 y Howland et al, 2001.

13 Véase: Cuenca, 2012.

14 Véase: Palacios, 2008. la maternidad no solo en el contexto de violaciones, sino también en casos en los que la relación sexual es consentida.

Otra cuestión que merece ser mencionada es la premisa falsa de que cualquier relación sexual practicada con una mujer con discapacidad es, en sí mismo, una violencia sexual. Muchas veces, con la intención de proteger a las mujeres con discapacidad de la amenaza de violencia sexual, sus familias, viéndolas como eternas niñas, no admiten la posibilidad de que deseen mantener relaciones sexuales, tratando cualquier señal de actividad sexual como una violencia, lo que anula su voluntad propia y las deshumaniza, como una demostración de discriminación (Macy, Parish y Wacker, 2008). Parece contradictorio que personas que estimen y se preocupen por ellas -como es el caso de un ambiente familiar sano- las discriminen. Sin embargo, no siempre la discriminación se demuestra a partir de su faceta más cruel, que pretende hacer daño intencionadamente, pudiendo suceder motivada por buenas intenciones. No obstante, independientemente de las intenciones que la originaron, la discriminación interseccional genera perjuicios.

Esto sucede más frecuentemente con las mujeres con discapacidad intelectual, debido a que la sociedad y el Derecho suelen utilizar una noción de consentimiento que no incluye a las personas con discapacidad intelectual o psicosocial, basada en la visión médico-rehabilitadora (Macy, Parish y Walker, 2008), además de que, socialmente, no se suele reconocer a las personas con discapacidad intelectual como individuos capaces de tener una relación de atracción y respeto basada en la voluntad propia (Bujan, 2016).

Doyle (2010) critica la ausencia de un debate en torno al consentimiento tanto en el discurso del modelo social de la discapacidad como en los autores que se dedicaron a renovar ese modelo. La autora propone fomentar ese debate cuestionando quienes son los que detienen el poder de decisión, las diferencias entre los géneros en la sociedad y la educación sexual ofrecida a las personas con discapacidad. Cabe destacar que no se debe relativizar la protección a las mujeres con discapacidad contra la violencia, pero esto exige un compromiso con el respeto a sus derechos sexuales en todos los ámbitos, incluso el de ejercer su libertad sexual.

Por todo lo expuesto, se concluye que los aspectos analizados, por sí mismos, no hacen que la mujer con discapacidad se encuentre en una posición de mayor riesgo de sufrir violencia sexual, pero pueden afectar o conducir las acciones delictivas de los agresores, debido al refuerzo de la discriminación interseccional que existe en torno a su imagen (Philips, 1996). Por ende, ellas no son, en sí mismas, más susceptibles a sufrir violencia sexual o sufren consecuencias negativas específicas debido a su discapacidad, sino que la discriminación interseccional se basa en estructuras sociales y aspectos culturales, institucionales y jurídicos que facilitan que este tipo de violencia ocurra con mayor predominancia en determinados grupos (Peláez y Villarino, 2016), entre ellos el de las mujeres con discapacidad.

\section{CONCLUSIONES}

La protección a los derechos sexuales de las mujeres con discapacidad, garantizándoles una vida libre de violencia y abuso sexual, exige comprender los matices que marcan la diferencia entre la idea de discriminación interseccional y de 
discriminación múltiple. Optar por un uso preciso del vocablo, nombrando específicamente los procesos discriminatorios interrelacionados como interseccionales, permite visibilizar las distintas formas de opresión que operan en un mismo individuo y concienciar la sociedad en torno a ello.

El uso de la expresión discriminación múltiple muchas veces ocurre sin que haya una reflexión sobre la razón por la que esa locución sería la más adecuada, sin cuestionar su uso en contextos en los que se podría optar por la palabra interseccional. El lenguaje importa, hay -o debe haber- una intencionalidad por detrás de la elección de un término en vez de otro, y no dedicarle la atención debida puede hacer posible no identificar ciertas causas y consecuencias de la discriminación.

En vista de ello, comprender el significado y la base teórica en torno a la expresión discriminación interseccional desde la perspectiva de distintos teóricos es el punto de partida para aplicarlo a contextos específicos como el de las mujeres con discapacidad víctimas de violencia sexual.

A su vez, proteger los derechos de las mujeres con discapacidad también requiere entender que no resaltarlas en el discurso de protección de los derechos de las mujeres es insuficiente para solucionar un problema que afecta directamente su libertad e igualdad. Las mujeres con discapacidad no logran acceder a ciertos derechos garantizados tanto a las demás mujeres como a los hombres con discapacidad, por lo que no destacarlas en ambos colectivos implica reproducir, aunque no intencionadamente, la discriminación.

Las mujeres con discapacidad experimentan un riesgo específico frente a la violencia sexual, derivado de la percepción negativa del agresor y de la sociedad sobre las mujeres con discapacidad, y entender que ellas no son, en sí mismas, más susceptibles o vulnerables a la violencia sexual permite enfocar el problema en el marco de la igualdad y la no discriminación y centrar las acciones de combate a la violencia sexual en el agresor, sin culpabilizar la víctima o revictimizarla.

Al pensar en soluciones y políticas para combatir la violencia sexual, también se debe tener siempre presentes el género y la discapacidad como elementos igualmente relevantes para la cuestión, pues priorizar uno de ellos significa tener una comprensión incompleta de su experiencia social como individuo, por lo que entender qué significa la discriminación interseccional es una herramienta esencial para proteger a las mujeres con discapacidad de la amenaza de violencia sexual.

\section{REFERENCIAS}

Almeida, Marlise. 2016. A banalização da violência contra as mulheres e a "cultura do estupro" no Brasil. Ágora. Belo Horizonte, 1: 126-131.

Arenas, Miriam. 2015. Una mirada interseccional a la violencia contra las mujeres con diversidad funcional. Oñati: Sociolegal series - violencia de género: intersecciones, 5: 367388.

Arnau, Soledad. 2005. Estudio La cara oculta de la violencia: la Violencia de Género contra la(s) Mujer(es) con disCapacidades. Castellón: Universitat Jaume I.

Ballester, María. 2013. Mujer y discapacidad. En: Serra,
Rosario. (org.) La discriminación múltiple en los ordenamientos jurídicos español y europeo, 155-198. Valencia: Tirant lo Blanch.

Barros, Lyvia y Jorge-Birol, Alline. 2007. Crime de estupro e sua vítima: a discriminação da mulher na aplicação da pena. Consultado el 09/06/2018. http://s3.amazonaws. com/academia.edu.documents/33961805

Basile, Kathleen y Smith, Sharon. 2011. Sexual Violence Victimization of Women: Prevalence, Characteristics, and the Role of Public Health and Prevention. American Journal of Lifestyle Medicine, 5: 407-417.

Benedet, Janine y Grant, Isabel. 2014. Sexual assault and the meaning of power and authority for women with mental disabilities. Consultado el 11/06/2018. http://link. springer.com/article/10.1007/s10691-014-9263-3

Bilge, Sirma y Collins, Patricia. 2016. Interseccionality: key concepts. Cambridge: Polity Press.

Brah, Avtar. 2012. Lineamientos generales en relación a la interseccionalidad. En: Chan, Jennifer; García, Sabina y Zapata, Martha (orgs.) La interseccionalidad en debate - Actas del Congreso Internacional Indicadores interseccionales y medidas de inclusión social en instituciones de educación superior. Berlín: Freie Universität.

Brownridge, D. 2006. Partner violence against persons with disabilities: prevalence, risks and explanations. Violence against women, 12: 805-822.

Bujan, Alejandro. 2016. Sexualidad sin barreras. Buenos Aires: Instituto Nacional contra la Discriminación, la Xenofobia y el Racismo - INADI.

Caballero, Isabel y Vales, Ana. 2012. Violencia: tolerancia cero. Apoyo psicosocial y prevención de la violencia de género en mujeres con discapacidad. Madrid: La Caixa.

Caballero, Isabel. 2016. La interseccionalidad de género y discapacidad a la luz de la Convención Internacional sobre los Derechos de las Personas con Discapacidad. En: Lorenzo, R. y Pérez Bueno, L. C. (dir.) La Convención Internacional sobre los derechos de las personas con discapacidad - 2006/2016: una década de vigencia, 93127. Madrid: Ediciones Cinca.

Campo, María Isabel. 2003. Aspectos de las relaciones afectivas y sexuales en personas con discapacidad intelectual. Consultado el 05/06/2018. http://www. informaciopsicologica.info/OJSmottif/index.php/ leonardo/article/view/361

Carignano, Florencia y Palacios, Agustina. 2012. Género, discapacidad y acceso a la justicia. En: Rosales, Pablo, Discapacidad, justicia y Estado: acceso a la justicia de personas con discapacidad, 61-68. Buenos Aires: Infojus.

Carrasco, María José; García, Pilar y Moya, Asunción. 2006. Discriminación en mujeres con discapacidad: una propuesta de prevención e intervención educativa. Educatio, 24: 99-122.

Carvalho, Maria Eulina; Dantas, Taísa y Silva, Jackeline. 2014. Entrelace entre gênero, sexualidade e deficiência: uma história feminina de rupturas e emponderamento. Revista Brasileira de Educação Especial, 4: 555-568.

Casique, Letícia y Furegato, Antônia. 2006. Violência contra mulheres: reflexões teóricas. Consultado el 20/07/2018. http://www.redalyc.org/html/2814/281421865018/

Cobo, Rosa. 2011. Hacia una nueva política sexual. Las 
mujeres ante la reacción patriarcal. Madrid: Los libros de la catarata.

Comité sobre los derechos de las personas con discapacidad de Naciones Unidas. 2016. Observación general 3: mujeres y niñas con discapacidad. Consultado el 11/06/2018.

Conde, Ángeles; Mun, Grace y Portillo, Inés. 2006. Mujer, discapacidad y violencia - El rostro oculto de la desigualdad. Madrid: Instituto de la Mujer.

Crenshaw, Kimberlé. 1989. Demarginalizing the Intersection of Race and Sex: A Black Feminist Critique of Antidiscrimination Doctrine, Feminist Theory and Antiracist Politics. Chicago: University of Chicago Legal Forum.

Crenshaw, Kimberlé. 2002. Documento para o encontro de especialistas em aspectos da discriminação racial relativos ao gênero. Revista de estudos feministas, 1: 78-99.

Cruz, María del Pilar. 2004. Mujeres con discapacidad y su derecho a la sexualidad. Política y Cultura, 22: 147-160.

Cruz, María del Pilar. 2012. Teoría feminista y discapacidad: un complicado encuentro en torno al cuerpo. Géneros Revista de investigación y divulgación sobre los estudios de género, 12: 51-71.

Cuenca, Patricia. 2012. El sistema de apoyo en la toma de decisiones desde la Convención Internacional sobre los Derechos de las Personas con Discapacidad: principios generales, aspectos centrales e implementación en la legislación española. Revista electrónica del Departamento de Derecho de la Universidad de La Rioja, 10: 61-94.

Dartnall, Elizabeth y Jewkes, Rachel. 2013. Sexual violence against women: The scope of the problem. Best Practice \& Research Clinical Obstetrics and Gynaecology, 27: 3-13.

Díaz, Elena. 2013. El reflejo de la mujer en el espejo de la discapacidad - La conquista de los derechos sexuales y reproductivos de las mujeres con discapacidad. Madrid: Cinca.

Doyle, Suzanne. 2010. The Notion of Consent to Sexual Activity for Persons with Mental Disabilities. Liverpool Law, 31: 111-135.

Eibach, Richard y Purdie-Vaughns, Valerie. 2008. Intersectional Invisibility: The Distinctive Advantages and Disadvantages of Multiple Subordinate-Group Identities. Consultado el 11/06/2018. https://www.researchgate. net/publication/225482308_Intersectional_Invisibility_ The_Distinctive_Advantages_and_Disadvantages_of_ Multiple_Subordinate-Group_Identities

Elman, Amy. 2005. Confronting the Sexual Abuse of Women with Disabilities. Harrisburg, PA: VAWnet, a project of the National Resource Center on Domestic Violence/ Pennsylvania Coalition Against Domestic Violence. Consultado el 08/06/2018. http://www.vawnet.org

Gesser, Marivet; Nuernberg, Adriano y Toneli, Maria. 2013. Constituindo-se sujeito na intersecção gênero e deficiência: relato de pesquisa. Psicologia em estudo, 3: 419-429.

Gobierno Vasco. 2012. Estudio sobre la especial vulnerabilidad de las mujeres con discapacidad víctimas de cualquier manifestación de violencia machista. http://www. once.es/new/plan-de-igualdad/estrategia-de-luchacontra-la-violencia-de-genero/Estudio\%20especial\%20 vulnerabilidad\%20mujeres\%20con\%20discapacidad\%20
-Gobierno\%20Vasco\%202012.pdf

Gomiz, María del Pilar. 2016. La sexualidad y la maternidad como factores adicionales de discriminación (y violencia) en las mujeres con discapacidad. Revista Española de Discapacidad (REDIS), 2: 123-142.

González Rams, Pilar. 2011. Las mujeres con discapacidad y sus múltiples desigualdades; un colectivo todavía insivibilizado en los Estados latinoamericanos y en las agencias de cooperación internacional. Congreso Internacional 1810-2010: 200 años de Iberoamérica. XIV Encuentro de Latinoamericanistas Españoles, 2737-2756.

Hanna, William y Rogovsky, Betsy. 2008. Mujer con discapacidad - suma de dos obstáculos. En: Barton, Len (comp.) Superar las barreras de la discapacidad - 18 años de Disability and Society, 51-67. Madrid: Morata.

Harnois, Catherine e Ifatunji, Mosi. 2011. Gendered measures, gendered models: toward an intersectional analysis of interpersonal racial discrimination, Ethnic and Racial Studies, 34: 6. Consultado el 18/05/2018. https:// doi.org/10.1080/01419870.2010.516836

Harpur, Paul. 2009. Sexism and racism, why not ableism? Calling for a Cultural Shift in the Approach to Disability Discrimination. Consultadoel27/05/2018. http://journals. sagepub.com/doi/abs/10.1177/1037969X0903400304

Hassouneh-Phillips, Dena y McNeff, Elizabeth. 2005. I Thought I was Less Worthy: Low Sexual and Body Esteem and Increased Vulnerability to Intimate Partner Abuse in Women with Physical Disabilities. Consultado el 02/06/2018. https://ohsu.pure.elsevier.com/en/ publications/i-thought-i-was-less-worsthy-low-sexualand-body-esteem-and-increa-2

Hlavka, Heather. 2014. Normalizing sexual violence: young women account for harassment and abuse. Gender \& Society, 28: 337-358.

Howland, Carol; Hugues, Rosemary y Nosek, Margaret. 2001. The investigation of abuse and women with disabilities going beyond assumptions. Violence against women, 7: 477-499.

Junta de Andalucía. Sin fecha. Mujeres con discapacidad y violencia sexual: guía para profesionales. Consejería para la igualdad y bienestar social. Consultado en 03/06/2018 http://www.juntadeandalucia.es/ igualdadybienestarsocial/opencms/system/bodies/ Personas_Discapacidad/Publicacion/Violencia_Sexual/ Violencia_sexual.pdf

La Barbera, Maria Caterina. 2016. Interseccionalidad, un "concepto viajero": orígenes, desarrollo e implementación en la Unión Europea. Interdisciplina, 4: 105-122.

Lama, Alejandra. 2013. Discriminación múltiple. Revista ADC, 1: 271-320.

Lledó, Eulalia. 2005. De lengua, diferencia y contexto. Pamplona: Gobierno de Navarra.

Lodrick, Zoe y Mason, Fiona. 2013. Psychological consequences of sexual assault. Best Practice \& Research Clinical Obstetrics and Gynaecology, 27: 27-37.

López, María. 2007. Discapacidad y género - estudio etnográfico sobre mujeres discapacitadas, 137-173. Consultado el 03/06/2018. https://dialnet.unirioja.es/ servlet/articulo?codigo $=2313642$

Macy, Rebecca; Parish, Susan y Wacker, Julia. 2008. Sexual Assault and Women with Cognitive Disabilities: Codifying 
Discrimination in the United States. Disability Policy Studies, 19: 86-94.

Makkonen, Timo. 2002. Multiple, compound and Intersectional discrimination: bringing the experiences of the most marginalized to the fore. Finlandia: Institute for Human Rights, Abo Akademi University.

Matsuda, Mari. 1991. Beside My Sister, Facing the Enemy: Legal Theory out of Coalition. Consultado el 02/06/2018. https://www.jstor.org/stable/1229035?seq=1\#page_ scan_tab_contents

Mays, Jennifer. 2006. Feminist disability theory: domestic violence against women with a disability. Disability \& Society, 1: 147-158.

Miller, Alice. 2000. Sexual but Not Reproductive: Exploring the Junction and Disjunction of Sexual and Reproductive Rights. Health and Human Rights, 2: 68-109.

Mogollón, María Esther. 2014. Cuerpos diferentes. Sexualidad y reproducción en mujeres con discapacidad. Consultado el 05/06/2018. http://repositoriocdpd.net:8080/ handle/123456789/393

Morris, Jenny. 1996. Encuentro con desconocidas: feminismo y discapacidad. Madrid: Narcea.

Moya, Asunción. 2009. La sexualidad en mujeres con discapacidad: perfil de su doble discriminación. Feminismo/s, 13: 133-151.

Organización Mundial de la Salud. 2013. Comprender y abordar la violencia contra las mujeres. Consultado el 20/07/2018. http://apps.who.int/iris/bitstream/ handle/10665/98821/WHO_RHR_12.37_spa. pdf?sequence $=1$

Palacios, Agustina. 2008. El modelo social de discapacidad: orígenes, caracterización y plasmación en la Convención Internacional sobre los Derechos de las Personas con Discapacidad. Madrid: Universidad Carlos III de Madrid, Instituto de Derechos Humanos "Bartolomé de las Casas".

Peláez, Ana y Villarino, Pilar. 2016. Informe sobre violencia de género hacia las mujeres con discapacidad a partir de la macroencuesta 2015. Consultado el 20/05/2018. http:// www.fundacioncermimujeres.es/sites/default/files/ informe_sobre_violencia_de_genero_2.pdf

Pelletier, Paola. 2014. La "discriminación estructural" en la evolución jurisprudencial de la Corte Interamericana de Derechos Humanos. Consultado el 05/06/2018. https:// revistas-colaboracion.juridicas.unam.mx/index.php/revinstituto-interamericano-dh/article/viewFile/8518/7615

Philips, Jennifer. 1996. Sexual assault, multiple disabilities and the law. Australian Feminist Law journal, 7: 157-162.

Pié, Assumpció y Riu, Carme. 2014. Violencia, mujer y diversidad funcional. La vulnerabilidad aumentada. Revista d'Intervenció Socioeducativa, 58: 45-64.

Platero, Lucas. 2014. Metáforas y articulaciones para una pedagogía crítica sobre la interseccionalidad. Consultado el 27/05/2018. http://www.quadernsdepsicologia.cat/ article/view/1219

Ramiro, Pedro. 2005. Igualdad y no discriminación de la mujer con discapacidad. En: Casado Muñoz, Raquel, Inclusión y no discriminación de la mujer con discapacidad, 39-43. Burgos: Universidad de Burgos.

Rey, Fernando. 2008. La discriminación múltiple, una realidad antigua, un concepto nuevo. Revista española de derecho constitucional, 28: 251-283.
Segato, Rita. 2017. La estructura de género y el mandato de violación. En: Caballero, Edith; de Santiago, Alejandra y González, Gabriela, Mujeres intelectuales: feminismo y liberación en América Latina y el Caribe, 299-332. Buenos Aires: CLACSO.

Serra, María Laura. 2017. Mujeres con discapacidad y situaciones de opresión. Desconstrucción feminista: desestabilizando las jerarquías de los dominios de poder. Tesis doctoral. Universidad Carlos III de Madrid. Consultado el 28/05/2018. https://e-archivo.uc3m.es/ handle/10016/24455

Villanueva, Rocío. 2006. Protección constitucional de los derechos sexuales y reproductivos. Revista IDH, 43: 391450. 